\title{
Qualidade de vida do profissional de enfermagem da atenção básica de saúde: revisão integrativa
}

RESUMO | Objetivou identificar a produção científica acerca da qualidade de vida da enfermagem que exerce suas atividades na atenção básica de saúde publicada em artigos nacionais, no período de 2010 a 2018, bem como, apresentar os enfoques principais levantados pelos autores dos estudos. Identificou-se a amostra de 345 artigos a partir da pesquisa na Biblioteca Virtual em Saúde (BVS) por meio dos descritores Qualidade de vida "and" Enfermagem, Enfermagem "and" Atenção Básica de Saúde e Qualidade de vida "and" Enfermagem "and" Atenção Básica de Saúde, dos quais 22 artigos se enquadraram nos critérios de inclusão. Embora alguns elencassem entre os descritores as palavras-chave pesquisadas, uma integra e cuidadosa leitura dos mesmos revelou que somente 5 destes artigos refletiam a relação da temática proposta. A análise dos artigos culminou na identificação de núcleos temáticos de onde emergiram as seguintes categorias: Adoecimento dos profissionais, relacionamento interpessoal, riscos ocupacionais, cargas de trabalho. Mediante a aplicação do método de pesquisa, constatou-se que são ínfimas as publicações atualizadas sobre a Qualidade de Vida do Profissional de Enfermagem da Atenção Básica de Saúde, indicando a necessidade de pesquisas que envolvam o tema.

Palavras-chaves: Enfermagem; Qualidade de Vida; Atenção Básica.

ABSTRACT | It aimed to identify the scientific production about the quality of life of nursing that carries out its activities in primary health care published in national articles, from 2010 to 2018, as well as to present the main approaches raised by the authors of the studies. The sample of 345 articles was identified from the research in the Virtual Health Library (VHL) through the descriptors Quality of life and Nursing, Nursing and Primary Health Care and Quality of life and Nursing and Primary Health Care, of which 22 articles met the inclusion criteria. Although some listed the keywords searched among the descriptors, a thorough and careful reading of them revealed that only 5 of these articles reflected the relationship of the proposed theme. The analysis of the articles culminated in the identification of thematic nuclei from which the following categories emerged: Illness of professionals, interpersonal relationships, occupational risks, workloads. Through the application of the research method, it was found that the updated publications on the Quality of Life of Nursing Professionals in Primary Health Care are negligible, indicating the need for research involving the theme.

Keywords: Nursing; Quality of Life; Primary Care.

RESUMEN | Su objetivo era identificar la producción científica sobre la calidad de vida de la enfermería que realiza sus actividades en atención primaria de salud publicadas en artículos nacionales, en el período de 2010 a 2018, así como presentar los principales enfoques planteados por los autores de los estudios. La muestra de 345 artículos se identificó a partir de la investigación en la Biblioteca Virtual en Salud (BVS) a través de los descriptores Calidad de vida y Enfermería, Enfermería y Atención Primaria de Salud y Calidad de vida y Enfermería y Atención Primaria de Salud, de los cuales 22 artículos cumplieron los criterios de inclusión. Aunque algunos enumeraron las palabras clave buscadas entre los descriptores, una lectura exhaustiva y cuidadosa de ellas reveló que solo 5 de estos artículos reflejaban la relación del tema propuesto. El análisis de los artículos culminó en la identificación de núcleos temáticos de los cuales surgieron las siguientes categorías: Enfermedades de los profesionales, relaciones interpersonales, riesgos laborales, cargas de trabajo. Mediante la aplicación del método de investigación, se descubrió que las publicaciones actualizadas sobre la Calidad de Vida de los Profesionales de Enfermería en Atención Primaria de Salud son insignificantes, lo que indica la necesidad de investigación sobre el tema.

Descriptores: Enfermería; Calidad de Vida; Atención Primaria.

\section{Paula Cristina de Sá Camargo Silveira}

Graduação em Enfermagem pela Universidade Federal de Goiás-Regional Jataí-Go

\section{Bruno Bordin Pelazza}

Enfermeiro. Pós-doutorando da Incubadora Tecnológica de Guarapuava - INTEG e docente do departamento de Enfermagem da Universidade Estadual do Centro-Oeste (UNICENTRO).

\section{Marcos André Mattos}

Enfermeiro. Doutor. Professor. Faculdade de Enfermagem. Universidade Federal de Goiás.

Recebido em: 09/03/2020

Aprovado em: 11/03/2020

\section{Meillyne Alves dos Reis}

Enfermeira. Doutoranda em Enfermagem pelaFaculdade deEnfermagem-Universidade Federal de Goiás. Docente do Curso de Enfermagem no Centro Universitário de Anápolis-Go/UniEVANGÉLICA.

\section{Lucila Pessuti Ferri}

Enfermeira. Doutoranda pelo Programa de Pós-Graduação em Enfermagem da Faculdade de Enfermagem - Universidade Federal de Goiás (UFG). Professora do Curso de Enfermagem UFG/Regional Jataí.

\section{Cácia Régia de Paula}

Enfermeira. Doutoranda em Enfermagem pela Faculdade de Enfermagem- Universidade Federal de Goiás. Professora Assistente da Universidade Federal de Goiás-Regional Jataí.
INTRODUÇÃO

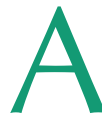
enfermagem remete à promoção e gestão do cuidado ao indivíduo e comunidade em geral. Os enfermeiros, técnicos e auxiliares de enfermagem compõem a equipe de enfermagem, prestam serviços nos mais variados contextos socioambientais e culturais ${ }^{(1)}$.

A Atenção Básica é considerada como porta de entrada preferencial do Sistema Único de Saúde (SUS), é ordenadora do serviço, efetiva no cuidado integralizado. Realiza promoção e proteção da saúde e prevenção de agravos ${ }^{(2)}$.

Segundo o grupo WHOQOL (1995), 
o conceito de qualidade de vida relaciona ao modo como o indivíduo percebe sua posição na vida em relação a cultura e seus valores, bem como sua posição em relação aos seus objetivos, expectativas, padrões e preocupações ${ }^{(3)}$.

Através do trabalho o ser humano busca ser aceito e estar inserido no meio social para ter suas necessidades, como indivíduo, sanadas. A qualidade de vida no trabalho é de extrema relevância, pois os reflexos do trabalho repercutem no cotidiano do sujeito. Alguns fatores podem acometer o profissional de enfermagem e comprometer sua qualidade de vida, promover o adoecimento e gerar $\operatorname{riscos}{ }^{(4)}$.

Frente ao panorama exposto, o fio condutor da pesquisa foi o questionamento: Qual é o conhecimento científico produzido no Brasil sobre a qualidade de vida da enfermagem na atenção básica, nos últimos anos? Com a finalidade de responder a tal questionamento, o presente estudo tem como objetivo identificar a produção científica acerca da qualidade de vida da enfermagem que exerce suas atividades na atenção básica de saúde publicada em artigos nacionais, no período de 2010 a 2018, bem como, apresentar os principais enfoques levantados pelos autores dos estudos.

\section{METODOLOGIA}

Trata-se de um estudo de revisão integrativa, realizado por meio de busca na Biblioteca Virtual em Saúde (BVS), nas bases de dados eletrônicas: Literatura Latino-Americana e do Caribe de Informação em Ciências da Saúde (LILACS) e Medical Literature Analysis and Retrieval System Online (MEDLINE).

A pesquisa bibliográfica é uma etapa fundamental em todo trabalho científico e influenciará em todas as etapas de uma pesquisa, dando o embasamento teórico em que se baseará o trabalho; as quais consistem: no levantamento, seleção, fichamento e análise das informações relacionadas à pesquisa, objetivando um resultado ${ }^{(5)}$.
A busca na base dados foi realizada utilizando as seguintes combinações de palavras-chave (unitermos) escolhidas mediante consulta aos Descritores em Ciências da Saúde (DeCS): Qualidade de vida "and" Enfermagem "and" Atenção Básica de Saúde. A seleção dos artigos foi realizada no mês de outubro de 2018, com base nos seguintes critérios de inclusão: artigos de periódicos on-line indexados nas bases de dados, com textos disponíveis na íntegra e em língua portuguesa, os quais deveriam refletir a temática, publicados no período compreendido entre 2010 e 2018. Excluíram-se: cartas, editoriais, teses, dissertações, revisões, capítulos de livros e demais textos não científicos.

Para a seleção dos artigos, realizou-se uma leitura prévia dos resumos das publicações selecionadas, por dois pesquisadores independentes, com objetivo de refinar a amostra por meio dos critérios de inclusão. Nos casos de divergência sobre a inclusão ou exclusão dos artigos, foi feita leitura minuciosa e discussão do estudo na íntegra, sendo que não havendo consenso, uma terceira pesquisadora decidiu a inclusão ou exclusão dos estudos.

A fase de análise dos artigos consistiu inicialmente na leitura dos estudos na íntegra, aplicação de instrumento para coleta de dados com os seguintes tópicos: título, autor(es), ano da publicação, fonte e principais resultados/achados da pesquisa. Os dados da coleta foram sintetizados em um quadro com resultado das pesquisas das palavras-chaves combinadas, posteriormente, categorizados para análise e discussão.

\section{RESULTADOS E DISCUSSÃO}

Identificou-se a amostra de 150 artigos a partir da pesquisa na Biblioteca Virtual em Saúde (BVS) por meio dos descritores Qualidade de vida "and" Enfermagem, dos quais 11 artigos se enquadraram nos critérios de inclusão. Embora alguns elencassem entre os descritores as palavras-chave pesquisadas, 
Figura 1. Fluxograma dos artigos e combinações de descritores utilizados. Guarapuava, PR, Brasil, 2019

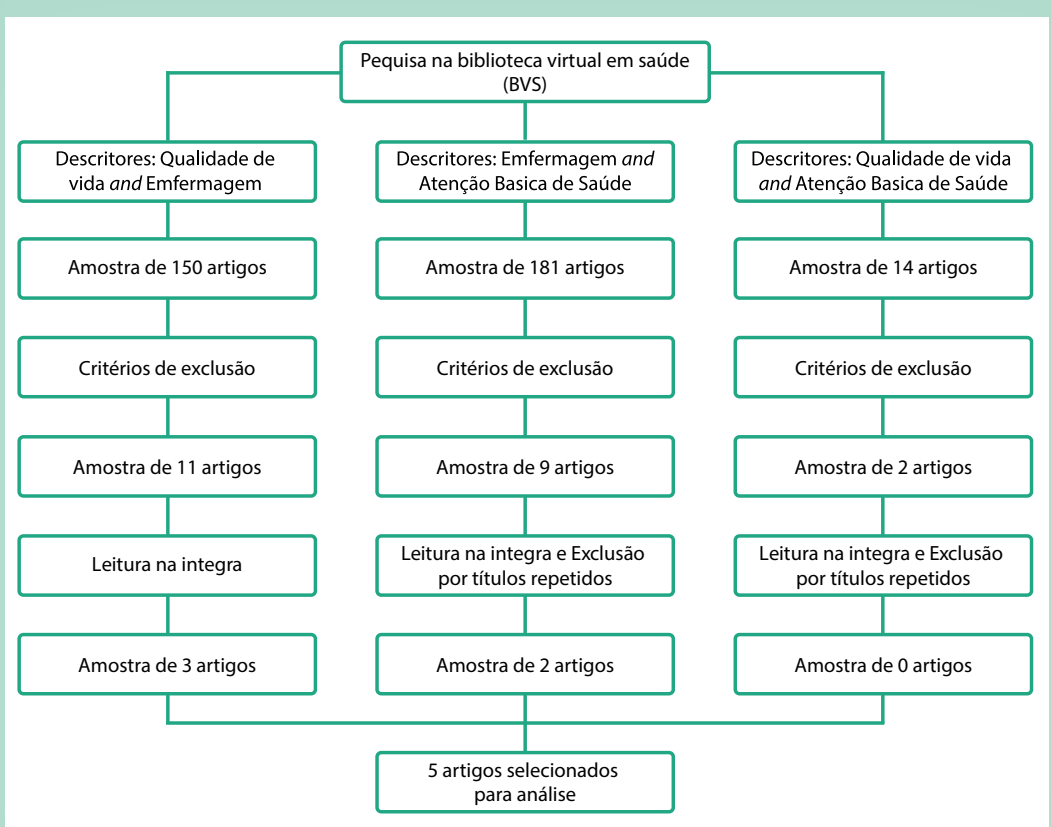

uma íntegra e cuidadosa leitura dos mesmos revelou que 03 destes artigos refletem a relação da temática proposta. Foi realizada uma segunda pesquisa, com a combinação dos descritores Enfermagem "and" Atenção Básica de Saúde, que resultou em uma amostra de 181 artigos, dos quais 09 se enquadraram nos critérios de inclusão. Após leitura minuciosa, exclusão por repetição de título, 02 artigos refletiam a temática. Uma terceira pesquisa foi realizada com a combinação dos descritores Qualidade de vida "and" Enfermagem "and" Atenção Básica de Saúde, resultando em 14 artigos, destes, 02 se enquadraram na temática, no entanto eram títulos repetidos das buscas anteriores, resultando em 0 artigos nesta associação de descritores. Totalizando o quantitativo de 05 artigos conforme o fluxograma da Figura 1:

Os artigos analisados foram distribuídos de acordo com o seguinte Quadro 1:

\section{Quadro 1. Estudos selecionados. Guarapuava, PR, Brasil, 2019}

\begin{tabular}{|c|c|c|c|c|}
\hline Título da Publicação & Autores & $\begin{array}{l}\text { Ano da } \\
\text { Publicação }\end{array}$ & $\begin{array}{l}\text { Veículo de } \\
\text { Publicação }\end{array}$ & Objetivos da Pesquisa \\
\hline $\begin{array}{l}\text { Aumento das cargas de } \\
\text { trabalho em técnicos de } \\
\text { enfermagem na atenção } \\
\text { primária à saúde no } \\
\text { Brasil }\end{array}$ & $\begin{array}{l}\text { Magda Duarte dos Anjos } \\
\text { Scherer; Neura Angélica de } \\
\text { Oliveira; Denise Elvira Pires de } \\
\text { Pires; Letícia de Lima Trindade; } \\
\text { Ana Sofia Resque Gonçalves; } \\
\text { Monica Vieira. }\end{array}$ & 2016 & $\begin{array}{l}\text { Revista Trabalho, } \\
\text { Educação e } \\
\text { Saúde. }\end{array}$ & $\begin{array}{l}\text { Analisar os principais aspectos } \\
\text { que fazem com que haja um aumento das } \\
\text { cargas de trabalho de técnicos de enferma- } \\
\text { gem da atenção primária à saúde da região } \\
\text { centro-oeste, norte sul do Brasil. }\end{array}$ \\
\hline $\begin{array}{l}\text { Síndrome de burnout em } \\
\text { enfermeiros na atenção } \\
\text { básica: repercussão na } \\
\text { qualidade de vida }\end{array}$ & $\begin{array}{l}\text { Ericka Silva Holmes; Sergio } \\
\text { Ribeiro dos Santos; Jamilton } \\
\text { Alves Faria; Maria Bernadete } \\
\text { de Sousa Costa. }\end{array}$ & 2014 & $\begin{array}{l}\text { Revista de Pes- } \\
\text { quisa Cuidado é } \\
\begin{array}{c}\text { Fundamental } \\
\text { online }\end{array}\end{array}$ & $\begin{array}{c}\text { Avaliar a repercussão da Síndrome de Bur- } \\
\text { nout na qualidade de vida dos enfermeiros } \\
\text { do município de João Pessoa-PB que atuam } \\
\text { na Atenção Básica }\end{array}$ \\
\hline $\begin{array}{l}\text { A produção científica } \\
\text { sobre a saúde do traba- } \\
\text { lhador de enfermagem }\end{array}$ & $\begin{array}{l}\text { Cecília Nogueira Valença; Lore- } \\
\text { na Mara Nóbrega de Azevêdo; } \\
\text { Aline Galúcio de Oliveira; } \\
\text { Samuel Sóstenes Araújo de } \\
\text { Medeiros; Fernanda Aparecida } \\
\text { Soares Malveira; Raimunda } \\
\text { Medeiros Germano.bbb }\end{array}$ & 2013 & $\begin{array}{l}\text { Revista de Pes- } \\
\text { quisa Cuidado é } \\
\begin{array}{c}\text { Fundamental } \\
\text { online }\end{array}\end{array}$ & $\begin{array}{l}\text { Identificar a produção científica a acerca da } \\
\text { saúde do trabalhador de enfermagem }\end{array}$ \\
\hline $\begin{array}{l}\text { Situação de trabalho dos } \\
\text { profissionais da } \\
\text { Estratégia Saúde da } \\
\text { Família em Ceará-Mirim }\end{array}$ & $\begin{array}{l}\text { Erika Simone Galvão Pinto; Re- } \\
\text { jane Maria Paiva de Menezes; } \\
\text { Tereza Cristina Scatena Villa. }\end{array}$ & 2010 & $\begin{array}{l}\text { Revista da esco- } \\
\text { la de enferma- } \\
\text { gem da USP }\end{array}$ & $\begin{array}{l}\text { Analisar a situação do trabalhador que } \\
\text { atua na Estratégia Saúde da Família em } \\
\text { Ceará-Mirim/Rio Grande do Norte. }\end{array}$ \\
\hline
\end{tabular}




\begin{tabular}{|l|l|c|c|c|}
\hline & $\begin{array}{l}\text { Márcia Batista Gil Nunes; } \\
\text { Raria Lúcia do Carmo Cruz } \\
\text { enfermeiros atuantes na }\end{array}$ & & & \\
$\begin{array}{l}\text { Atenção à saúde da } \\
\text { família }\end{array}$ & $\begin{array}{l}\text { Robazzi; Fábio de Souza Terra; } \\
\text { Regina Célia Gollner Zeitoune; } \\
\text { lara Aparecida de Oliveira } \\
\text { Secco. }\end{array}$ & \multirow{2}{*}{2010} & $\begin{array}{l}\text { Revista Enfer- } \\
\text { magem UERJ }\end{array}$ & $\begin{array}{l}\text { Descrever os riscos ocupacionais dos enfer- } \\
\text { meiros de Volta Redonda/RJ que atuam na } \\
\text { Rede Básica de Saúde }\end{array}$ \\
\hline
\end{tabular}

A análise dos artigos culminou na identificação de núcleos temáticos de onde emergiram as seguintes categorias: Adoecimento dos profissionais, Relacionamento interpessoal, Riscos ocupacionais e Cargas de trabalho.

\section{Adoecimento dos profissionais}

$\mathrm{O}$ adoecimento dos profissionais de enfermagem é algo decorrente de inúmeros fatores que fazem parte da sua realidade. Existem várias formas de adoecimento, entre elas o físico, que pode ser resultado de excessos de esforços, movimentos repetitivos, alta demanda de trabalho, falta de tempo para descanso, a forma em que o trabalho se encontra distribuído. O adoecimento psicológico é outra forma que é recorrente no ambiente da Atenção Básica de Saúde. O sofrimento do trabalhador que não sente vontade em ir trabalhar, que não sabe se seu adoecimento é físico ou mental, tudo isto é reflexo da sua rotina diária, da falta de pessoal , consequentemente, sobrecarga de outros, falta de recursos materiais e mesmo da forma em que a população também adoecida, cobra o atendimento dos profissionais de enfermagem, o contato é direto, impossível não se abalar ${ }^{(6)}$.

A síndrome do esgotamento profissional, conhecida como síndrome de burnout, tem grande relevância entre os profissionais da área da saúde, pois afeta diretamente a qualidade de vida desta classe de trabalhadores. A síndrome acontece quando, após o desgaste, o trabalhador desiste do trabalho e não há mais satisfação em realiza-lo ${ }^{(7)}$.

Apoio e a devida proteção social para a enfermagem desenvolver suas atribuições são raros, apesar de que, para exercer o trabalho de enfermagem, é neces-

\section{S6}

sário ter boa saúde mental e física, a fim de se prevenir acidentes e adoecimentos derivados das suas atividades ${ }^{(8)}$.

O ser humano tem cada vez mais realizado atividades complexas em seu dia a dia e buscado por excelência em tudo que realiza, as responsabilidades emergidas de suas profissões estão acarretando doenças emocionais, psicológicas e físicas. Por ser o profissional da área da saúde um promotor do cuidado, é importante que os mesmos busquem a promoção da própria saúde e bem como, o controle ou prevenção de seus adoecimentos. Umas das medidas contra o estresse do trabalhador que atua na área da saúde são: a musicoterapia, exercícios de relaxamento para a hora de descanso, massagens $\operatorname{etc}^{(9)}$.

as seguintes

categorias:

Adoecimento dos profissionais, Relacionamento interpessoal, Riscos ocupacionais e Cargas de trabalho.
Relacionamento interpessoal

A relação profissional-profissional e profissional-usuário gera diversos conflitos diariamente. A humanização que é cobrada na forma de atendimento para com o próximo, deve ser a mesma no relacionamento entre os profissionais que atuam na Atenção Básica de Saúde e do usuário para os profissionais. A forma de organização do trabalho inadequada ocasiona em acúmulo de tarefas e exaustão dos profissionais, acarretando desgaste no contato com o usuário ${ }^{(6)}$.

O convívio entre pessoas diferentes, no mesmo ambiente de trabalho, com atribuições interdependentes, pode tornar-se um lugar competitivo, fazendo com que haja necessidade de socialização, cordialidade, empatia e ética, ou seja é imprescindível que se tenha uma doação de todos profissionais em prol de uma qualidade de vida melhor ${ }^{(10)}$. 


\section{Riscos ocupacionais}

$\mathrm{O}$ autocuidado juntamente com o cuidar do próximo, para o profissional de enfermagem, se torna exaustivo e gerador de estresse, quando ocorre alteração nesse processo, que acontece de forma natural(8).

Os riscos ocupacionais, que acometem os trabalhadores de enfermagem, são na maioria das vezes passíveis de prevenção. Esses riscos estão sendo estudados, no entanto, ainda fazem muitas vítimas por passarem despercebidos aos profissionais.

Em um estudo(11) realizado no município de Volta Redonda-RJ, de 2008 a 2009, com enfermeiros que atuam na rede básica de saúde, emergiram seis categorias de riscos ocupacionais: físico, de acidente de trajeto, ergonômico, mecânico, psicossocial e biológico. Os riscos ocupacionais são campo de pesquisa da saúde do trabalhador no Brasil, com abordagem para o ambiente seguro para trabalhador, livre de riscos ambientais e com prevenção de fatores que alterem estas condições de qualidade no trabalho.

O profissional de enfermagem está constantemente exposto a riscos em seu ambiente de trabalho na Atenção Básica de Saúde em decorrência das atribuições desempenhadas e do contato direto com o usuário. Estar bem consigo mesmo, em suas melhores condições, já contribui para a minimização de acidentes ou ausência de erros nesse ambiente de trabalho.

\section{Cargas de trabalho}

A sobrecarga de trabalho, mão de obra sem qualificação e escassa, absenteísmo, jornada dupla de trabalho são alguns fatores que afetam a área da saúde e da enfermagem, no Brasil ${ }^{(12)}$.

Ao corpo do trabalhador associa-se a carga de trabalho, que podem manifestar-se nos fatores biológicos e psíquicos, repercutindo em degradação do estado físico e emocional(6).

Para os membros da Estratégia Saúde da Família (ESF) da Atenção Primária a Saúde, é estabelecido uma carga horaria obrigatória de 40 horas semanais de trabalho. No entanto, a realidade do profissional de enfermagem, muitas vezes, é uma associação do trabalho dele na Atenção Básica com jornadas em hospitais. $\mathrm{O}$ fator salarial é um dos motivos para que isso aconteça. Outro fator relevante, é o fato de a enfermagem ser composta em sua maioria por pessoas do sexo feminino provedoras de suas famílias.

Um estudo ${ }^{(13)}$ realizado em Ceará-Mirim, Rio Grande do Norte em 2007, cujo objetivo era analisar as situações de trabalho vivenciadas pelos profissionais de saúde da ESF desta mesma região, evidenciou que es- tes profissionais consideravam suas remunerações financeiras insuficientes para atingirem uma qualidade de vida. Consequência disso, buscam em outro vínculo empregatício o complemento para suas necessidades.

\section{CONCLUSÃO}

Mediante a aplicação do método de pesquisa, constatou-se a necessidade de novos estudos acerca da Qualidade de Vida do Profissional de Enfermagem da Atenção Básica de Saúde. Este estudo apresenta como limitação a pequena quantidade de estudos que trata o tema. Espera-se que esta pesquisa venha contribuir para o surgimento de mais pesquisas subsequentes a respeito da temática. Cabe ressaltar a importância de estudos que busquem conhecer e estimar a Qualidade de Vida do Profissional de Enfermagem da Atenção Básica de Saúde, de modo a proporcionar um ambiente seguro para o profissional de enfermagem, livre de riscos de saúde e ambientais prevenindo fatores que alterem as condições de qualidade no trabalho, bem como a valorização deste em termos de remuneração financeira.

Esta pesquisa não recebeu financiamento específico de nenhuma agência de fomento dos setores públicos, comercial ou sem fins lucrativos.

\section{Referências}

1. Conselho Federal de Enfermagem (BR). Código de ética de Enfermagem. Resolução Cofen n. ${ }^{\circ}$ 564/2017, de 06 de novembro de 2017. 2. Ministério da Saúde (BR)'. Portaria n. 2436 de 21 de setembro de 2017. Brasília: Diário da República Federativa do Brasil, 2017.

3. Fleck MPA. 0 instrumento de avaliação de qualidade de vida da Organização Mundial da Saúde (WHOQOL-100): características e perspectivas. Revista ciência e saúde coletiva. 2000; 5(1).

4. Lobo LMGA, Oliveira FBM. Qualidade de vida dos profissionais de enfermagem: uma revisão integrativa de literatura. Revista ciência e saberes. 2015.

5. Amaral JJF. Como fazer uma pergunta bibliográfica. Fortaleza, janeiro de 2007.

6. Scherer MDA, et al. Aumento das cargas de trabalho em técnicos de enfermagem na Atenção primária à saúde no Brasil. Revista Trabalho, Educação e Saúde. 2016; 14:89-104.

7. Holmes ES, et al. Síndrome de burnout em enfermeiros na atenção básica: repercussão na qualidade de vida. Revista de Pesquisa Cuidado é Fundamental On-line. 2014; 6(4):1384-1395.

8. Valença $\mathrm{CN}$, et al. A produção científica sobre a saúde do traba-
Ihador de enfermagem. Revista de Pesquisa Cuidado é Fundamental On-line. 2013; 5(5):52-60.

9. Ribeiro RP, et al. 0 adoecer pelo trabalho na enfermem: uma revisão integrativa. Revista da escola de enfermagem da USP. 2012; 46(2):495-504.

10. Cardoso CG, Silva LO. A importância do relacionamento interpessoal no ambiente de trabalho. Revista Multidisciplinar da Faculdade de Ciências Biológicas e da Saúde da Unigran. 2014; 8(2):24-33.

11. Nunes MBG, et al. Riscos ocupacionais dos enfermeiros atuantes na Atenção à saúde da família. Revista Enfermagem UERJ. 2010; 18(2):204-209.

12. Magalhães AMM, et al. Carga de trabalho da equipe de enfermagem e segurança do paciente - estudo com método misto na abordagem ecológica restaurativa. Revista Latino-Americana de Enfermagem. 2013.

13. Pinto ESG, Menezes RMP, Villa TCS. Situação de trabalho dos profissionais da Estratégia Saúde da Família em Ceará-Mirim. Revista da escola de enfermagem da USP. 2010; 44(3):657-664. 